\title{
Enhanced performance of heterogeneous networks through full-duplex relaying
}

\author{
Hirley Alves ${ }^{1,2^{*}}$, Mehdi Bennis ${ }^{1}$, Richard Demo Souza ${ }^{2}$ and Matti Latva-aho ${ }^{1}$
}

\begin{abstract}
In this article, we focus on the uplink of a heterogeneous network composed of a macrocell and an underlaid femtocell. We address the problem of the interference caused by the macro user (MU) on the femtocell [femto base station (FBS) and users]. The femtocell is composed of an user, a femto relay, which can be seen as another FBS or a dedicated relay, and an FBS. We assume a full-duplex relay (FDR) node which is able to transmit and receive simultaneously while suffering from residual self-interference. We focus on the performance of the femtocell according to different positions of the MU user. We derive closed-form expressions for the outage probability and spectral efficiency (throughput) taking into account the self-interference of the FDR node. Moreover, we assume that the nodes are able to apply successive interference cancellation on the MU signal. Our results show that FDR can considerably enhance the performance of the femtocell, even in the presence of strong self-interference at the FDR, allowing the femtocell to operate in a high rate regime.
\end{abstract}

\section{Introduction}

In recent years, the demand for higher data rates increased considerably and new technologies have been able to respond to the demand by efficiently reusing the spectrum. Moreover, it is known that in a wireless network an effective way to increase capacity is by making transmitter and receiver closer [1]. Femtocells appear as a less expensive alternative to meet that demand by increasing indoor coverage and spectral efficiency. Nevertheless, due to reduced spectrum availability the femtocell will have to support spectrum sharing techniques. The coexistence in the same spectrum of macro and femtocells increases the spectral efficiency but poses several challenges, such as additional interference between macro and femtocells [1] which becomes a major concern when these networks do not cooperate [2]. Therefore, for optimum performance the femtocells have to control the interference induced on the macro user $(M U)$, as well as to manage the interference caused by the $M U[3,4]$.

The interference conditions in a femtocell network are directly related to the access policy used at the femto base station (FBS), which can be categorized as closed or open

*Correspondence: halves@ee.oulu.fi

${ }_{1}^{1}$ Centre for Wireless Communications (CWC), University of Oulu, Oulu, Finland

${ }^{2}$ Federal University of Technology - Paran (UTFPR), Curitiba, Brazil
$[5,6]$. In the case when the femtocell employs a closed policy, the femtocell subscribers are only served by the FBS. On the other hand, in an open access policy the femtocell may accept connections of non-subscriber users. A closed access policy may reduce the femtocell performance due to interference from MUs, while an open access policy increases the number of handovers for MUs and requires more resources of the femtocell. For instance, in [7] the transmission capacity of open and closed access policies is evaluated in the uplink (UL) of a heterogeneous network. Moreover, it is shown that in the UL open access is preferable since a reduction in interference is obtained. Conversely, as shown in [6], in the downlink (DL) the closed access policy is preferred by the femto users (FUs) which contrasts with the preference of the MUs. Chu et al. $[8,9]$ investigate the DL of a heterogeneous network where macro and femtocells share the spectrum. The femtocell operates in a subset of the resource allocated to the macrocell which reduces the interference caused by the femtocell. Chu et al. [8] developed a resource allocation scheme, while in [9] an outage probability analysis is derived. In [10], resource allocation is investigated based on the opportunistic reuse of the macrocell spectrum. The results show that by increasing performance of the FUs, the $M U$ suffers extra interference which limits its performance.

\section{是 Springer}

2012 Alves et al.; licensee Springer. This is an Open Access article distributed under the terms of the Creative Commons Attribution License (http://creativecommons.org/licenses/by/2.0), which permits unrestricted use, distribution, and reproduction in any medium, provided the original work is properly cited. 
Moreover, in this article we assume that the macrocell network is operating in frequency division duplex (FDD) mode while the small cells are (re)-using the macrocell UL resources and operate in FD mode, which means that unused resources by the macrocell can locally be reused within small cells where some nodes can transmit and receive simultaneously in an FD fashion. The use of FD nodes can potentially increase the capacity of small cells, while adhering to the cross-tier interference constraint imposed by the macrocell. Note that the proposed scheme solves the multiplexing loss of HD schemes, the DL/UL switching point optimization required in the classical time division duplex (TDD) [11] underlay at UL FDD. Thus, the macrocell operation is oblivious to the presence of underlaid small cells, which are opportunistic in nature.

Differently form other works in TDD underlay femtocells [12-14], we analytically investigate the performance of a single femtocell, considering that the femtocell nodes are able to employ selective cooperative protocols. Moreover, we assume a single femtocell composed by an FU (source - S), an FDR, which can be another FBS or a dedicated node, and the intended destination $(D)$ or access point. The relay helps the communication between the FU and the FBS by forwarding the source message. The practical relay is assumed to operate in the FD mode, which means that it is able to transmit and receive simultaneously, and hence is subject to self-interference from the transmitted to the received signal. Next we address the related work in cooperative and full-duplex schemes.

\subsection{Related work on FDR}

Cooperative communication is an alternative to achieve spatial diversity even with single antenna devices [15]. In a cooperative scheme a relay helps the source to communicate with the destination, and its behavior is dictated by the cooperative protocols such as amplify-and-forward and the decode-and-forward (DF), or their variants selective and incremental $[15,16]$.

In the Selective-DF (SDF), the relay forwards the source message only if that is error free. Nevertheless, with half duplex (HD) radios the cooperation suffers a multiplexing loss, once the relay listen in a first slot (time/frequency) and then retransmits the message in a second slot. Several solutions have been proposed trying to overcome the problem of the HD constraint as in $[17,18]$ and references therein. On the other hand, incremental cooperative protocols, such as incremental-DF (IDF), can overcome the spectral inefficiency of HD cooperation through the exploitation of a return channel between nodes $[15,16,19]$. In the IDF protocol, the relay only cooperates with the source if a retransmission is requested by the destination, so that it is not necessary to previously allocate a slot for the relay operation.
Another way to overcome the spectral inefficiency of the HD cooperative schemes is through cooperative FD relaying, which does not suffer from multiplexing loss and can achieve a higher capacity than HD cooperative protocols [16]. Probably the most known FD schemes are multihop (FD-MH) and block Markov encoding (FD-BM). The FD-MH scheme is the simplest relaying technique, while FD-BM is the best known performance achieving FD method. However, FD-BM is quite complex to be implemented in practice. Moreover, perfect isolation (ideal) between transmitted and received signals is often not possible. In practice, isolating the transmitted and received signals is not straightforward, once the transmitted power is normally much larger than the received power [16]. Therefore, practical FD schemes have been proposed in which it is considered that there is a power leakage between transmitted and received signals, also known as loop interference or self-interference [20-22]. For instance, Riihonen et al. [20] show that practical FD-MH is feasible even if the relay faces strong selfinterference. Moreover, it is also shown that FD-MH relaying enhances capacity when compared to the HD scheme. In addition, similar conclusions were obtained in [21-24]. In [25], a practical FD system is proposed and the authors show that the self-interference can be attenuated by more than $39 \mathrm{dBs}$ through transmit and receive antenna separation.

\subsection{Proposed scheme}

As aforementioned, differently form other works in TDD underlay femtocells [12-14], we analytically investigate the performance of a single femtocell, considering that the femtocell nodes are able to employ selective cooperative protocols. Moreover, the relay is a practical FD relay, and to the best of the authors' knowledge the usage of FD nodes within the femtocell cannot be found in the literature. We analyze the femtocell performance in terms of outage probability and spectral efficiency. We also assume that the relay and the FBS are able to apply successive interference cancellation (SIC) techniques [26]. We analyze the performance considering mainly two scenarios regarding the position of the $M U$ : (i) the $M U$ is far from the femtocell, and therefore does not interfere on the femtocell communication; and (ii) the $M U$ is close to the femtocell, and the interference has to be taken into account. The main contributions of this article are twofold: (i) derivation of some closed form outage probability expressions considering SIC in the FD scenario; (ii) performance analysis of FD relaying in a heterogeneous network. Furthermore, our results show that FD relaying can considerably enhance performance within the femtocell, allowing the femto nodes to operate in a higher rate regime when compared to the $M U$. 
The rest of this article is organized as follows. Section 2 presents the system model. Section 3 introduces the outage and spectral efficiency analysis. In Section 4, some numerical results are presented. Finally, Section 5 concludes the article.

\section{System model}

Consider the UL of a heterogeneous network composed of a femtocell and a macrocell. The latter is characterized by one $M U$ communicating with the macro base station (MBS) in the UL, while the former is composed of a source $(S)$ or FU, communicating to an FBS or destination $(D)$. The communication is established through the help of a relay $(R)$. We also assume the $M U$ to be at the border of the macro cell. There are at least two possible scenarios regarding the position of the $M U$ :

- The $M U$ is far from the femtocell, and therefore the femtocell can operate normally on the same resource, considering that the $M U$ does not interfere with the femtocell, and its signal is seen as noise.

- The $M U$ is near the femtocell and therefore interferes on the femtocell communication.

Figure 1 illustrates both scenarios: Figure 1a considers the femtocell alone once the interference caused by the $M U$ can be seen as noise because the $M U$ is far from the femtocell; Figure 1b considers a heterogeneous network and emphasizes the interference links. Notice that we assume a practical FD relay which suffers from self-interference (loop interference). The self-interference signal is the power leakage from the transmitted to the received signal. We assume that the self-interference link is dominated by the scattering component of the channel, once the line of sight component is considerably reduced by antenna isolation. The self-interference may actually represent the residual interference after the application of an interference cancellation technique [22,24,25]. Moreover, we assume that all channels are subject to quasi-static Rayleigh fading, with zero mean and unit variance. We assume perfect channel state information at the receivers. The noise is assumed to be of the complex additive white Gaussian noise type with variance $N_{0} / 2$ per dimension.

We consider that both $R$ and $D$ are able to employ SIC on the $M U$ interference signal. Therefore, $R$ and $D$ see a multiple access channel and the rate regions and individual outage probabilities can be defined as in $[26,27]$, respectively. As shown in [28], SIC can successfully be applied in cellular networks, while an example of application on a femtocell scenario can be found in [29,30], where HD nodes are able to cancel the interference through SIC.

In this article, we employ the path loss models introduced in [31-33]. Considering an indoor femtocell deployment, we assume that the path loss is given by

$$
\mathrm{PL}_{i j}(\mathrm{~dB})=39.676+20 \log _{10}\left(d_{i j}\right)+q W+X_{\sigma}
$$

where the first term corresponds to the free-space path loss, $d_{i j}$ is the distance between transmitter $i$ and receiver $j, W$ is the wall partition loss, which we assume to be $5 \mathrm{~dB}, q$ is the number of walls between the transmitter and receiver (assumed $q=1$ except for the $S-D$ link, where

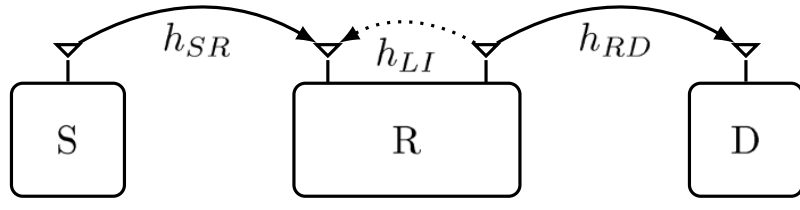

(a) First Scenario

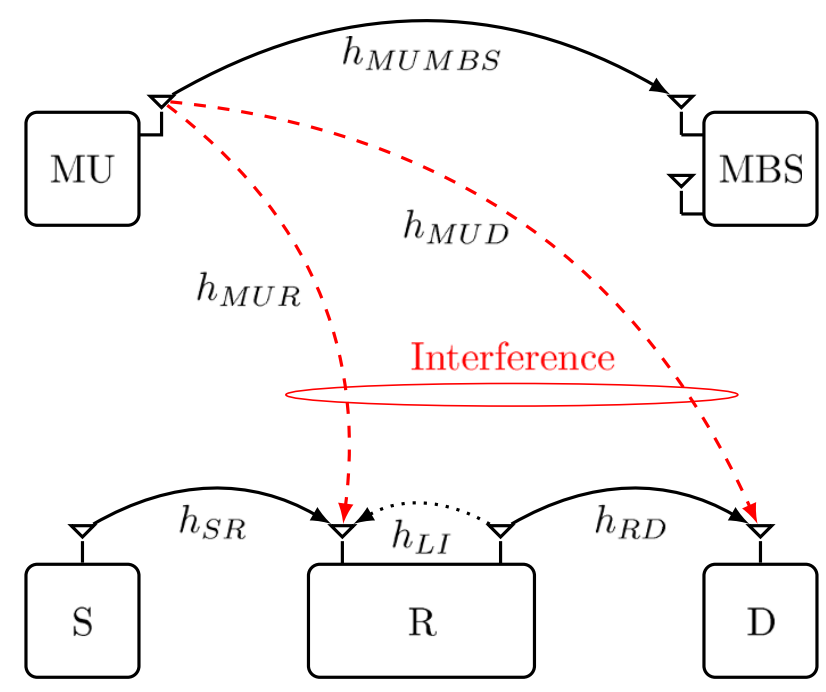

(b) Second Scenario

Figure 1 In the first scenario, the distance of $M U$ is much larger than $d_{S R}$ so that the interference caused by the $M U$ can be seen as noise. However, in second case, the $M U$ is much closer to $R$ and $D$, thus interference cannot be neglected. 
we assume $q=2$ ) and $X_{\sigma}$ is a zero mean Gaussian distributed random variable (in $\mathrm{dB}$ ) with standard deviation $\sigma=6 \mathrm{~dB}[14]$.

The outdoor-to-indoor path loss model is

$$
\mathrm{PL}_{i j}(\mathrm{~dB})=39.676+20 \log _{10}\left(d_{i j}\right)+q W+W^{\prime}+X_{\sigma},
$$

where $W^{\prime}$ is the outdoor penetration loss that is assumed equal to $10 \mathrm{~dB}$, and $X_{\sigma}$ is a zero mean Gaussian distributed random variable with standard deviation $\sigma=8 \mathrm{~dB}$ [14].

\section{$2.1 \quad$ FD-MH relaying}

We assume an FD-MH relaying scheme operating under the SDF protocol [15], in which $S$ broadcasts a message and $R$ forwards it to $D$ only if it was able to decode the message error free. Moreover, in our scenario we assume that the $M U$ may interfere on the femtocell according to its positioning. Therefore, we can write the signal received at $R$ as

$$
\begin{aligned}
y_{R}= & \sqrt{P_{S} \kappa_{S R}} h_{S R} x_{S}+\sqrt{P_{M U} \kappa_{M U R}} h_{M U R} x_{M U} \\
& +\sqrt{P_{R}} h_{L I} x_{R}+n_{R}, \\
y_{D}= & \sqrt{P_{R} \kappa_{R D}} h_{R D} x_{R}+\sqrt{P_{M U} \kappa_{M U D}} h_{M U D} x_{M U}+n_{D},
\end{aligned}
$$

where $h_{i j}$ represents the fading channel coefficients between the nodes $i \in\{S, R, M U\}$ and $j \in\{R, D\}$, while $h_{L I}$ is the complex fading coefficient of the loop interference [22] and $\kappa_{i j}$ is the inverse of the path loss between nodes $i$ and $j$. The transmit power of each node is $P_{i}$ where $i \in\{S, R, M U\}$. Notice that when the $M U$ is far from the femtocell we can assume that $\kappa_{M U R}$ and $\kappa_{M U D} \rightarrow 0$, and therefore the $M U$ does not interfere on the femtocell. Moreover, the additive complex Gaussian noises are represented by the terms $n_{R}$ and $n_{D}$. In addition, the source message is represented by $x_{S}$, while the message transmitted by the relay $x_{R}$ is a function of the received signal from the source. Moreover, $E\left[\left|x_{S}\right|^{2}\right]=E\left[\left|x_{R}\right|^{2}\right]=1$, where $E[\cdot]$ is the mathematical expectation. Notice that the transmitted relay signal is a delayed version of the source signal, and we assume that the delay is long enough to guarantee that the transmitted and received signals at the relay are uncorrelated. Notice that once we assume that the $S-D$ link has worse large-scale fading condition than the $S-R$ and $R-D$ links, it is reasonable to assume that $D$ will see the $S-D$ signal as noise instead of interference $[20,23,34]$.

The instantaneous and average signal-to-noise ratio (SNR) of each link are

$$
\gamma_{i j}=\frac{P_{i} \kappa_{i j}\left|h_{i j}\right|^{2}}{N_{0}}
$$

and

$$
\bar{\gamma}_{i j}=\frac{P_{i} \kappa_{i j} E\left[\left|h_{i j}\right|^{2}\right]}{N_{0}}
$$

while the instantaneous and average SNR of the selfinterference link can be written, respectively, as $\gamma_{L I}=$ $\frac{P_{R} \Phi\left|h_{L I}\right|^{2}}{N_{0}}$ and $\bar{\gamma}_{L I}=\frac{P_{R} \Phi E\left[\left|h_{L I}\right|^{2}\right]}{N_{0}}$. We assume that the self-interference channel suffers a constant attenuation which is represented by $\Phi$. Moreover, as shown in [25], in a practical scenario it is possible to attenuate the selfinterference by as much as $40 \mathrm{~dB}$ according to the set of techniques employed, as for instance spatial antenna separation.

Since the fading envelope is Rayleigh distributed, the probability density function of the instantaneous SNRs is [35]

$$
f_{\gamma_{i j}}\left(\gamma_{i j}\right)=\frac{1}{\gamma_{i j}} \exp \left(-\frac{\gamma_{i j}}{\overline{\gamma_{i j}}}\right), \quad \gamma_{i j} \geq 0 .
$$

The instantaneous signal-to-interference plus noise ratio (SINR) seen by the receivers are

$$
\gamma_{R}=\frac{\gamma_{S R}}{\gamma_{M U R}+\gamma_{L I}+1}
$$

and

$$
\gamma_{D}=\frac{\gamma_{R D}}{\gamma_{M U D}+1}
$$

Notice that the relay suffers interference from the $M U$ and from itself, while $D$ is interfered only by the $M U$.

\section{$2.2 \mathrm{HD}-\mathrm{MH}$ relaying}

We also consider the case of HD-MH in order to show the performance implications of operating the relay in the FD or HD modes. In the case of HD-MH two time slots are necessary per source message, as the relay listens first and then retransmits the signal to $D$. Since we assume the SDF protocol, $R$ only retransmits if the received signal was decoded error free. Thus, the received signal at $D$ can be written as in (4), while the received signal at $R$ is given by

$$
y_{R}=\sqrt{P_{S} \kappa_{S R}} h_{S R} x_{S}+\sqrt{P_{M U} \kappa_{M U R}} h_{M U R} x_{M U}+n_{R},
$$

where the notation follows the one used above. Moreover, the SNR and SINR are defined just as above, with the only difference that in HD-MH there is no self-interference at the relay.

Finally, in the case of a direct transmission (DT), in which $S$ communications directly with $D$ without the help of the relay, the received signal can be written as

$$
y_{D}=\sqrt{P_{S} \kappa_{S D}} h_{S D} x_{S}+\sqrt{P_{M U} \kappa_{M U D}} h_{M U D} x_{M U}+n_{D} .
$$

For DT the SINR is simply $\gamma_{D}^{d i r}=\frac{\gamma_{S D}}{\gamma_{M U D}+1}$. 


\section{Outage and spectral efficiency analysis}

In this section, we characterize the outage and spectral efficiency of FD-MH and HD-MH relaying schemes described in Section 2.

\subsection{DT}

First, we characterize the rate region of the DT scheme supposing that $D$ applies SIC, according to [26,27]. Thus ${ }^{\mathrm{a}}$

$$
\begin{aligned}
\mathcal{R} & \leq \log \left(1+\gamma_{S D}\right), \\
\mathcal{R}_{M U} & \leq \log \left(1+\gamma_{M U D}\right), \\
\mathcal{R}+\mathcal{R}_{M U} & \leq \log \left(1+\gamma_{S D}+\gamma_{M U D}\right),
\end{aligned}
$$

where $\mathcal{R}$ is the information rate used by $S$, while $\mathcal{R}_{M U}$ is the information rate used by the $M U$. The decoding events related to this region are

1. (a) Both messages are decoded;

2. (b) Only $S$ is decoded and therefore $M U$ is in outage;

3. (c) Only $M U$ is decoded and therefore $S$ is in outage;

4. (d) Unable to decode, both $S$ and $M U$ are in outage.

Figure 2a illustrates the MAC rate region and the related decoding events. Notice that in order to determine the outage probability of the DT scheme we can first determine the probabilities of events (c) and (d) as illustrated in Figure 2a. The integration regions are illustrated in Figure $2 \mathrm{~b}$ and note that we split the region representing event (d) into two sub-regions namely $\mathbf{B}$ and $\mathbf{C}$, while the event (c) is represented by $\mathbf{A}$ in Figure $2 b$. Thus, with help of Figure $2 b$ we can determine the integration limits for each region and finally we can write the outage probability of the DT scheme as
Notice that the underbraces in (15) correspond to the integration regions of Figure $2 \mathrm{~b}$.

Furthermore, if $\bar{\gamma}_{M U D}=\bar{\gamma}_{S D}$, then (15) reduces to [27]:

$$
\begin{aligned}
\mathcal{O}_{\text {dir }}= & -\frac{2^{-\mathcal{R}} \exp \left(\frac{1}{\bar{\gamma}_{S D}}\right)}{\bar{\gamma}_{S D}}\left(\exp \left(-\frac{2^{\mathcal{R}}}{\bar{\gamma}_{S D}}\right) \bar{\gamma}_{S D}\right. \\
& \left.+\left(2^{\mathcal{R}}-1\right) \exp \left(-\frac{2^{\mathcal{R}_{M U}+\mathcal{R}}}{\bar{\gamma}_{S D}}\right)\left(2^{\mathcal{R}}\left(2^{\mathcal{R}_{M U}}-1\right)+\bar{\gamma}_{S D}\right)\right) .
\end{aligned}
$$

If the $M U$ is far away from the femtocell we can assume that there is no interference at $D$, and therefore SIC is not necessary. Consequently, we can rewrite the outage probability of the DT simply as $\mathcal{O}_{\mathrm{dir}}=1-\exp \left(-\frac{2^{\mathcal{R}}-1}{\bar{\gamma}_{S D}}\right)$.

Another metric of interest is the spectral efficiency, which is also known as throughput. The spectral efficiency is defined as the average rate of correct information transfer and is given in bits/s/Hz. Therefore, the spectral efficiency for of $S-D$ link is given by:

$$
T_{\text {dir }}=\mathcal{R} \cdot\left(1-\mathcal{O}_{\text {dir }}\right)
$$

\subsection{HD relaying}

Next, we derive the outage probability of the HD-MH relaying scheme. Following the same rationale used to derive the rate regions and the individual outage probability of the $S-D$ link, we can obtain, through appropriate substitutions, the individual outage probabilities of the $S-R$ and $R-D$ links, $\mathcal{O}_{S R}$ and $\mathcal{O}_{R D}$, respectively. Thus, at the $S-R$ link we take (15) and substitute $\bar{\gamma}_{M U D}, \bar{\gamma}_{S D}$ by

$$
\begin{aligned}
& \mathcal{O}_{\text {dir }}=\mathcal{O}_{\text {dir }}=\operatorname{Pr}[(c)]+\operatorname{Pr}[(d)] \\
& =\underbrace{\operatorname{Pr}\left[\mathcal{R}>C\left(\gamma_{S D}\right), \mathcal{R}_{M U}<C\left(\frac{\gamma_{M U D}}{1+\gamma_{S D}}\right)\right]}_{\mathbf{A}}+\underbrace{\operatorname{Pr}\left[\mathcal{R}>C\left(\gamma_{S D}\right), \mathcal{R}_{M U}>C\left(\frac{\gamma_{M U D}}{\gamma_{S D}+1}\right)\right]}_{\mathbf{B}} \\
& +\underbrace{\operatorname{Pr}\left[\mathcal{R}<C\left(\gamma_{S D}\right), \mathcal{R}>C\left(\frac{\gamma_{S D}}{\gamma_{M U D}+1}\right), \mathcal{R}+\mathcal{R}_{M U}>C\left(1+\gamma_{S D}+\gamma_{M U D}\right)\right]}_{\mathbf{C}} \\
& =\int_{0}^{2^{\mathcal{R}}-1} \int_{\left(2^{\left.\mathcal{R}_{M U}-1\right)\left(\gamma_{S D}+1\right)}\right.}^{\infty} f_{\gamma_{S D}}\left(\gamma_{S D}\right) \cdot f_{\gamma_{M U D}}\left(\gamma_{M U D}\right) d \gamma_{M U D} d \gamma_{S D} \\
& +\int_{0}^{2^{\mathcal{R}}-1} \int_{0}^{\left(2^{\mathcal{R}_{M U}}-1\right)\left(\gamma_{S D}+1\right)} f_{\gamma_{S D}}\left(\gamma_{S D}\right) \cdot f_{\gamma_{M U D}}\left(\gamma_{M U D}\right) d \gamma_{M U D} d \gamma_{S D} \\
& +\int_{2^{\mathcal{R}}-1}^{2^{\mathcal{R}_{M U}\left(2^{\mathcal{R}}-1\right)}} \int_{\frac{\gamma_{S D}}{2^{\mathcal{R}}-1}-1}^{2^{\left(\mathcal{R}+\mathcal{R}_{M U}\right)}-1-\gamma_{S D}} f_{\gamma_{S D}}\left(\gamma_{S D}\right) \cdot f_{\gamma_{M U D}}\left(\gamma_{M U D}\right) d \gamma_{M U D} d \gamma_{S D} \\
& =1-\frac{\exp \left(-\frac{2^{\mathcal{R}}\left(2^{\mathcal{R}_{M U}}-1\right)}{\bar{\gamma}_{M U D}}+\frac{1-2^{\mathcal{R}}}{\bar{\gamma}_{S D}}\right) \bar{\gamma}_{M U D}}{\bar{\gamma}_{M U D}-\bar{\gamma}_{S D}}+\frac{\exp \left(-\frac{2^{\mathcal{R}_{M U}-1}}{\bar{\gamma}_{M U D}}-\frac{2^{\mathcal{R}_{M U}\left(2^{\mathcal{R}}-1\right)}}{\bar{\gamma}_{S D}}\right) \bar{\gamma}_{M U D}}{\bar{\gamma}_{M U D}-\bar{\gamma}_{S D}} \\
& -\frac{\left(2^{\mathcal{R}}-1\right) \exp \left(\frac{1}{\bar{\gamma}_{M U D}}-\frac{2^{\mathcal{R}_{M U}}\left(\left(2^{\mathcal{R}}-1\right) \bar{\gamma}_{M U D}+\bar{\gamma}_{S D}\right)}{\bar{\gamma}_{S D} \bar{\gamma}_{M U D}}\right) \bar{\gamma}_{M U D}}{\left(2^{\mathcal{R}}-1\right) \bar{\gamma}_{M U D}+\bar{\gamma}_{S D}}-\frac{\exp \left(-\frac{2^{\mathcal{R}}-1}{\bar{\gamma}_{S D}}\right) \bar{\gamma}_{S D}}{\left(2^{\mathcal{R}}-1\right) \bar{\gamma}_{M U D}+\bar{\gamma}_{S D}} .
\end{aligned}
$$


(a)

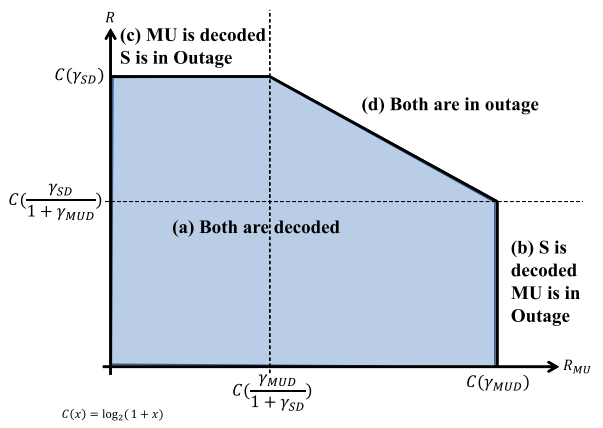

(b)

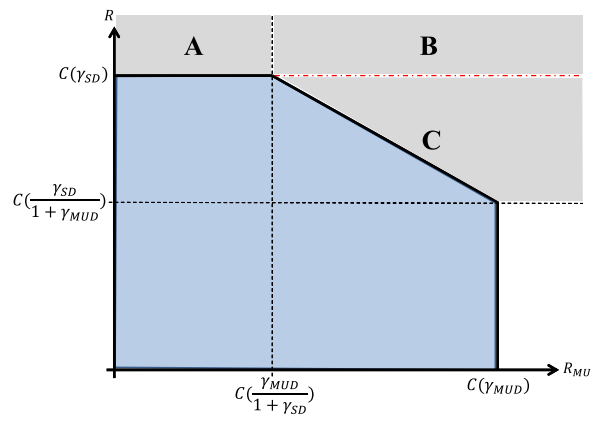

Figure 2 Rate and integration regions. (a) Illustrates the MAC rate regions and we emphasize four possible decoding events. (b) Shows the integration regions used to determined the outage probability of the DT transmission.

$\bar{\gamma}_{M U R}, \bar{\gamma}_{S R}$, respectively. At the $R-D$ link we substitute only $\bar{\gamma}_{S D}$ by $\bar{\gamma}_{R D}$.

Now, we can define the overall outage probability of the HD-MH scheme as

$$
\mathcal{O}_{H D-M H}=\mathcal{O}_{S R}+\left(1-\mathcal{O}_{S R}\right) \cdot \mathcal{O}_{R D} .
$$

Notice that the communication is successful only if both links are not in outage. Moreover, similar to the direct scheme when the $M U$ is far away from the femto cell we can, respectively, rewrite the outage probabilities of the $S-R$ and $R-D$ links as $\mathcal{O}_{S R}=1-\exp \left(-\frac{2^{\mathcal{R}}-1}{\bar{\gamma}_{S R}}\right)$ and $\mathcal{O}_{R D}=1-\exp \left(-\frac{2^{\mathcal{R}}-1}{\bar{\gamma}_{R D}}\right)$.

The overall spectral efficiency of the HD-MH scheme can be defined as

$$
T_{\mathrm{HD}-\mathrm{MH}}=\frac{\mathcal{R}}{2}\left(1-\mathcal{O}_{\mathrm{HD}-\mathrm{MH}}\right) .
$$

The factor $\frac{1}{2}$ present in the spectral efficiency of the $\mathrm{HD}-\mathrm{MH}$ scheme is a consequence of the HD constraint of the cooperative HD schemes with selective protocols.

\subsection{FDR}

The rate regions for the FD-MH scheme for the $M U$ and $S$ signals are given by [26]

$$
\begin{aligned}
\mathcal{R} & \leq \log \left(1+\frac{\gamma_{S R}}{\gamma_{L I}+1}\right), \\
\mathcal{R}_{M U} & \leq \log \left(1+\frac{\gamma_{M U R}}{\gamma_{L I}+1}\right), \\
\mathcal{R}+\mathcal{R}_{M U} & \leq \log \left(1+\frac{\gamma_{S R}+\gamma_{M U R}}{\gamma_{L I}+1}\right) .
\end{aligned}
$$

The outage probability of the $S-R$ link when the $M U$ is near to the femtocell could not be derived in closed-form. Moreover, as $D$ is an HD device, and therefore the individual outage probability of the $R-D$ link of FD-MH can be derived in the same manner as in HD-MH.
When the $M U$ is distant from the femtocell, then SIC is not necessary. Therefore, $R$ suffers only self-interference. By making use of some standard transformations [36] we can derive the outage probability of the $S-R$ link in closedform as

$$
\begin{aligned}
\mathcal{O}_{S R} & =\operatorname{Pr}\left[\log \left(1+\gamma_{R}\right)<\mathcal{R}\right]=\operatorname{Pr}\left[\frac{\gamma_{S R}}{\gamma_{L I}+1}<2^{\mathcal{R}}-1\right] \\
& =\int_{0}^{2^{R}-1} f_{\gamma_{R}}\left(\gamma_{R}\right) d \gamma_{R}=1-\frac{\exp \left(-\frac{2^{\mathcal{R}}-1}{\bar{\gamma}_{S R}}\right) \cdot \bar{\gamma}_{S R}}{\bar{\gamma}_{S R}+\left(2^{R}-1\right) \cdot \bar{\gamma}_{L I}},
\end{aligned}
$$

where $\operatorname{Pr}[\theta]$ is the probability of event $\theta$. Similar to the HD scheme, the outage at the $R-D$ link when the $M U$ is far from the femtocell reduces to

$$
\begin{aligned}
\mathcal{O}_{R D} & =\operatorname{Pr}\left[\gamma_{R D}<2^{\mathcal{R}}-1\right]=\int_{0}^{2^{R}-1} f_{\gamma_{R D}}\left(\gamma_{R D}\right) d \gamma_{R D} \\
& =1-\exp \left(-\frac{2^{\mathcal{R}}-1}{\bar{\gamma}_{R D}}\right) .
\end{aligned}
$$

Thus, the overall outage probability $\mathcal{O}_{F D-M H}$ can be written as in (18), but using the $\mathcal{O}_{S R}$ for the case of an FD relay given in (23). Moreover, when we assume that $M U$ is distant we can write the overall outage probability as

$$
\mathcal{O}_{\mathrm{FD}-\mathrm{MH}}=1-\frac{\exp \left(-\frac{\left(2^{\mathcal{R}}-1\right) \cdot\left(\bar{\gamma}_{S R}+\bar{\gamma}_{R D}\right)}{\bar{\gamma}_{S R} \cdot \bar{\gamma}_{R D}}\right) \bar{\gamma}_{S R}}{\bar{\gamma}_{S R}+\left(2^{\mathcal{R}}-1\right) \cdot \bar{\gamma}_{L I}} .
$$

Based on the overall outage probabilities, we can define the overall spectral efficiency:

$$
T_{\mathrm{FD}-\mathrm{MH}}=\mathcal{R} \cdot\left(1-\mathcal{O}_{\mathrm{FD}-\mathrm{MH}}\right) .
$$

Notice that the great advantage of FD relaying is that it is possible to achieve the same maximum spectral efficiency as DT, which is twice the maximum spectral efficiency achieved by HD. However, as the FD relay suffers from 
self-interference, it is not clear if the net results are positive or not. In the next section, we numerically investigate this issue.

\section{Numerical results}

In this section, we present some numerical results considering the position of the $M U$. Moreover, we analyze the performance of the femtocell for different values of self-interference attenuation and information rates. We perform a semi-analytic analysis of the heterogeneous network studied since we do not derive a closed-form expression for the $S-R$ link of the FD-MH scheme when the $M U$ is near the femtocell, instead we employ Monte Carlo simulations in which we consider at least $10^{6}$ channel realizations for each SNR value. Furthermore, we recall that we derived closed-form expressions for all other links. We assume that the FBS has a radius of $r_{f}=20 \mathrm{~m}$, while the MBS has a radius of $r_{\mathrm{m}}=400 \mathrm{~m}$. The FU (or $S$ ) and the FD relay are positioned over a straight line towards $D$, so that $d_{S R}=d_{R D}=20 \mathrm{~m}$, while $d_{S D}=40 \mathrm{~m}$. We assume that the $S-D$ link faces two walls, therefore in this case $q=2$. We also consider that the carrier frequency is $2.3 \mathrm{GHz}$, and without loss of generality we assume that $N_{0}=1$. The other path loss parameters are described in Section 2. Moreover, we assume that the femtocell nodes have the same power $P$. Thus, considering a fair comparison in terms of total power, since the cooperative schemes employ a total power equal to $P_{T}=2 P$, we assume that in the DT scheme the source uses $P_{S}=2 P$, and also that the $M U$ always transmits with $P_{M U}=2 P$.
Figure 3 presents the overall outage probability as a function of the average SNR at the $S-D$ link $\bar{\gamma}_{S D}$. We assume different levels of self-interference attenuation at the relay $\Phi=[-10 ;-20 ;-40] \mathrm{dB}[25]$, two different positions for the $M U$ which are given by $d_{M U R}=$ $d_{M U D}=[10 ; 50] \mathrm{m}$, and that the femtocell operates in a high rate regime in which $\mathcal{R}=4 \mathrm{bits} / \mathrm{s} / \mathrm{Hz}$, while the $M U$ information rate is $\mathcal{R}_{M U}=2 \mathrm{bits} / \mathrm{s} / \mathrm{Hz}$ [14]. We can see from the figure that even with low attenuation levels the FD-MH scheme outperforms the DT in the low SNR regime. According to Duarte and Sabharwal [25], who implemented a practical FD node, larger levels of interference attenuation can be obtained in practice through simple antenna separation techniques with a small distance between antennas, e.g., $39 \mathrm{~dB}$ at $20 \mathrm{~cm}$. The authors also show that larger cancellation gains are achievable in practice. Moreover, such dimensions are feasible in practice when we address the femtocell as a home base station once multiple antenna settings would require similar dimensions $[37,38]$. Nevertheless, as we show in Figure 3, with only $40 \mathrm{~dB}$ attenuation it is already possible to achieve gains around $6 \mathrm{~dB}$ in the high SNR regime, performing close to the HD-MH scheme in terms of outage probability. Furthermore, we conclude from the figure that in terms of outage probability the HD-MH outperforms the FD-MH scheme. Nonetheless, as we show next, this conclusion does not hold when spectral efficiency is considered, since as aforementioned, selective HD cooperative schemes are spectrally inefficient $[17,18]$.

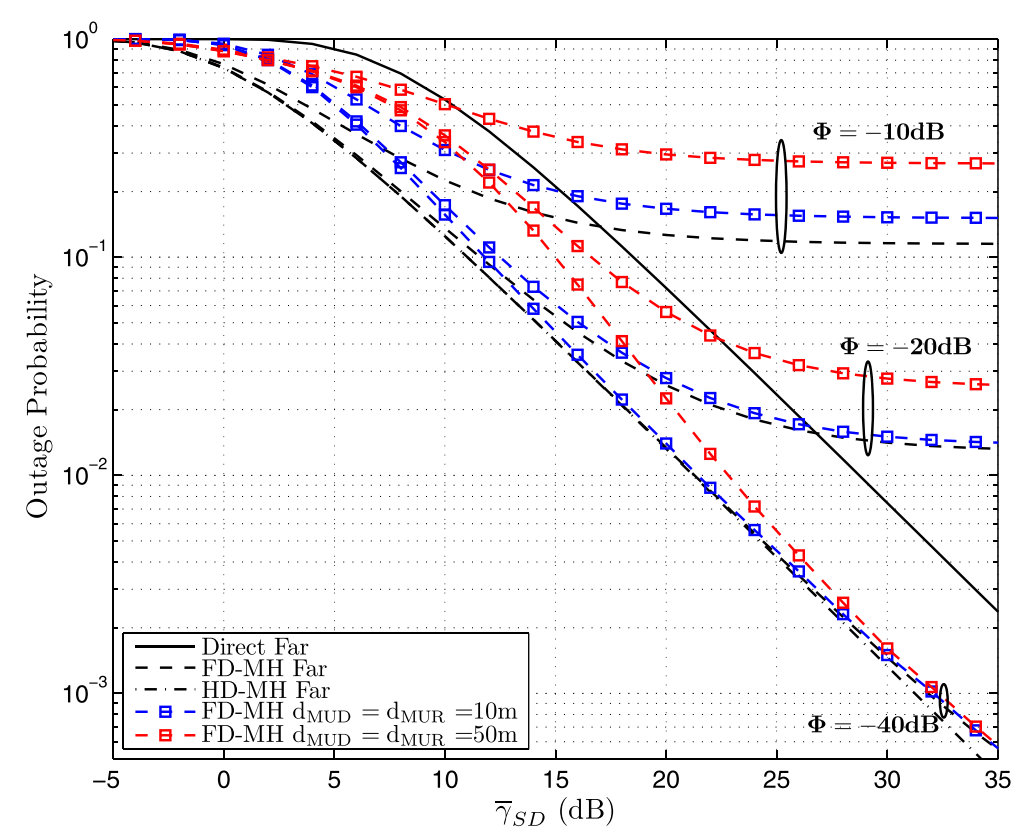

Figure 3 Outage probability as a function of the $\bar{\gamma}_{S D}$, considering different levels of self-interference attenuation at the relay $(\Phi=[-10 ;-20 ;-40] \mathrm{dB})$ and positioning of the $M U d_{M U R}=d_{M U D}=[10 ; 50] \mathrm{m}$. 
Figure 4 shows the femtocell spectral efficiency as a function of the $\bar{\gamma}_{S D}$, where $\mathcal{R}=4 \mathrm{bits} / \mathrm{s} / \mathrm{Hz}$ and $\mathcal{R}_{M U}=$ $2 \mathrm{bits} / \mathrm{s} / \mathrm{Hz}$. We also assume different levels of selfinterference attenuation, and as discussed above, larger values of attenuation increase performance. Notice that if the relay node suffers too much from self-interference (low attenuation level), the performance of the FD-MH is degraded and it is not possible to achieve the maximum spectral efficiency in the high SNR regime. Nevertheless, full performance can already be obtained with $40 \mathrm{~dB}$ of interference cancellation. Another relevant conclusion that can be obtained from Figures 3 and 4 is that at medium to high SNR regime the proximity of the $M U$ to the femtocell favors the performance of $S$, once the interference signal is stronger and therefore can easily be removed [35]. On the other hand, at an intermediate distance the $M U$ signal is weaker and harder to be removed as we can see from the figures. However, note that in the low SNR region those conclusions do not hold, as the rate used by $S$ is too high and the interference cannot successfully be removed by means of SIC. Furthermore, as a benchmark, we can determine the performance of $S$ when $M U$ does not interfere at all on the femtocell due to its positioning. Considering that, we can see that FD-MH considerably outperforms HD-MH and DT schemes in the whole SNR range. Notice that the multiplexing loss due to the HD constraint can be observed in Figure 4 in the high SNR region, where the DT and the FD-MH schemes achieve full performance (the spectral efficiency is equal to $\mathcal{R}$ ) while the HD-MH scheme can achieve at most $\mathcal{R} / 2$.

Next we analyze the impact of the $M U$ positioning on the performance of the femtocell, where for the case of the FD relay $\Phi=[-40] \mathrm{dB}$. We present in Figure 5 the femtocell spectral efficiency as a function of the $d_{M U R}$, which is the distance between $M U$ and the relay. We assume that $M U$ and relay are over a straight line, and that $d_{M U D}=$ $\sqrt{d_{R D}^{2}+d_{M U R}^{2}}$. In the figure, we also show the spectral efficiency achieved by the three methods if the $M U$ is far from the femtocell (thus, in these cases the spectral efficiency is not a function of $d_{M U D}$ and is represented by straight lines). Supporting the conclusion obtained in Figure 4 we can see that the performance of the femtocell decreases if the $M U$ is at an intermediate position. In the case of $\bar{\gamma}_{S D}=15 \mathrm{~dB}$, Figure $5 \mathrm{a}$ shows that in the case of FD-MH and $\mathrm{HD}-\mathrm{MH}$ the interference from the $M U$ is either successfully removed or negligible if the $M U$ is very close to the femtocell $\left(d_{M U D}<20 \mathrm{~m}\right)$ or if the $M U$ is very far from the femtocell $\left(d_{M U D}>300 \mathrm{~m}\right)$. Note that the DT is more susceptible to the $M U$ interference than FD-MH and $\mathrm{HD}-\mathrm{MH}$, showing an important advantage in terms of robustness of the relaying methods. However, if the channel conditions within the femtocell are better then the effect of the $M U$ is greatly reduced. Figure $5 \mathrm{~b}$ shows that when $\bar{\gamma}_{S D}=25 \mathrm{~dB}$, the $M U$ interference does not affect at all the relaying methods, while the DT is still affected.

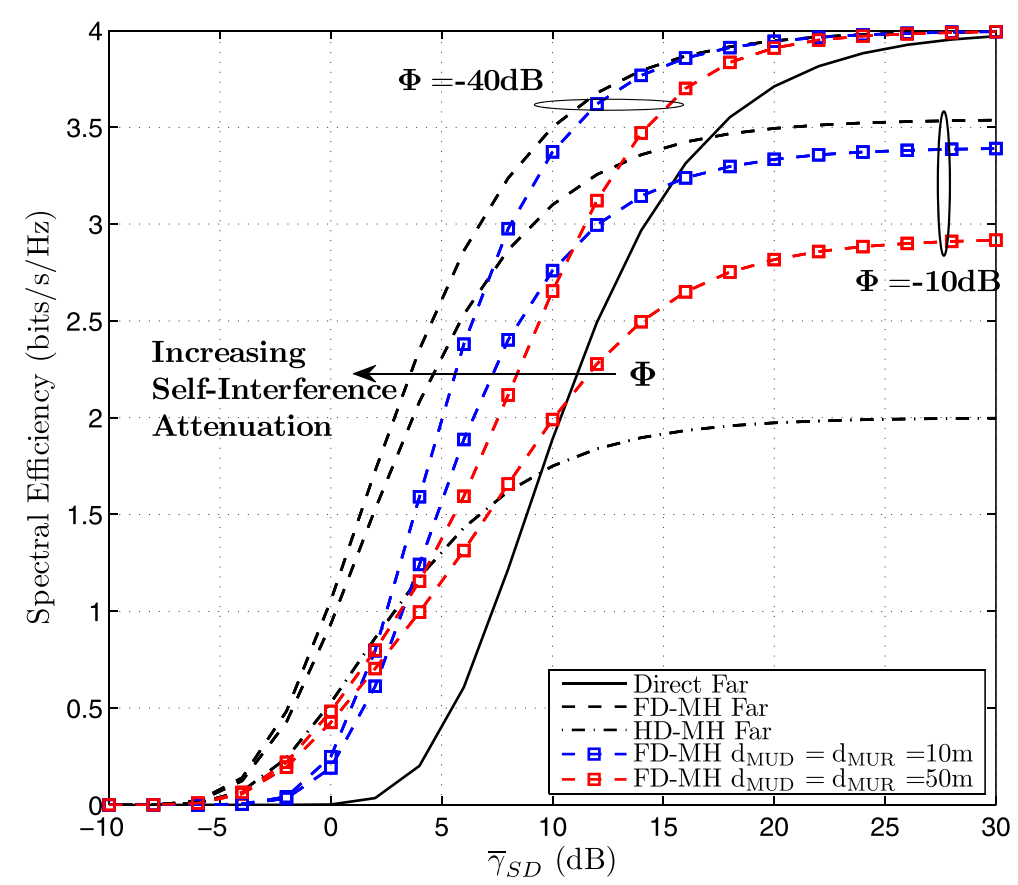

Figure 4 Spectral efficiency as a function of the $\bar{\gamma}_{S D}$, considering different levels of self-interference attenuation at the relay $\Phi=[-10 ;-40] \mathrm{dB}$, and positioning of the $M U d_{M U R}=d_{M U D}=[10 ; 50] \mathrm{m}$. 

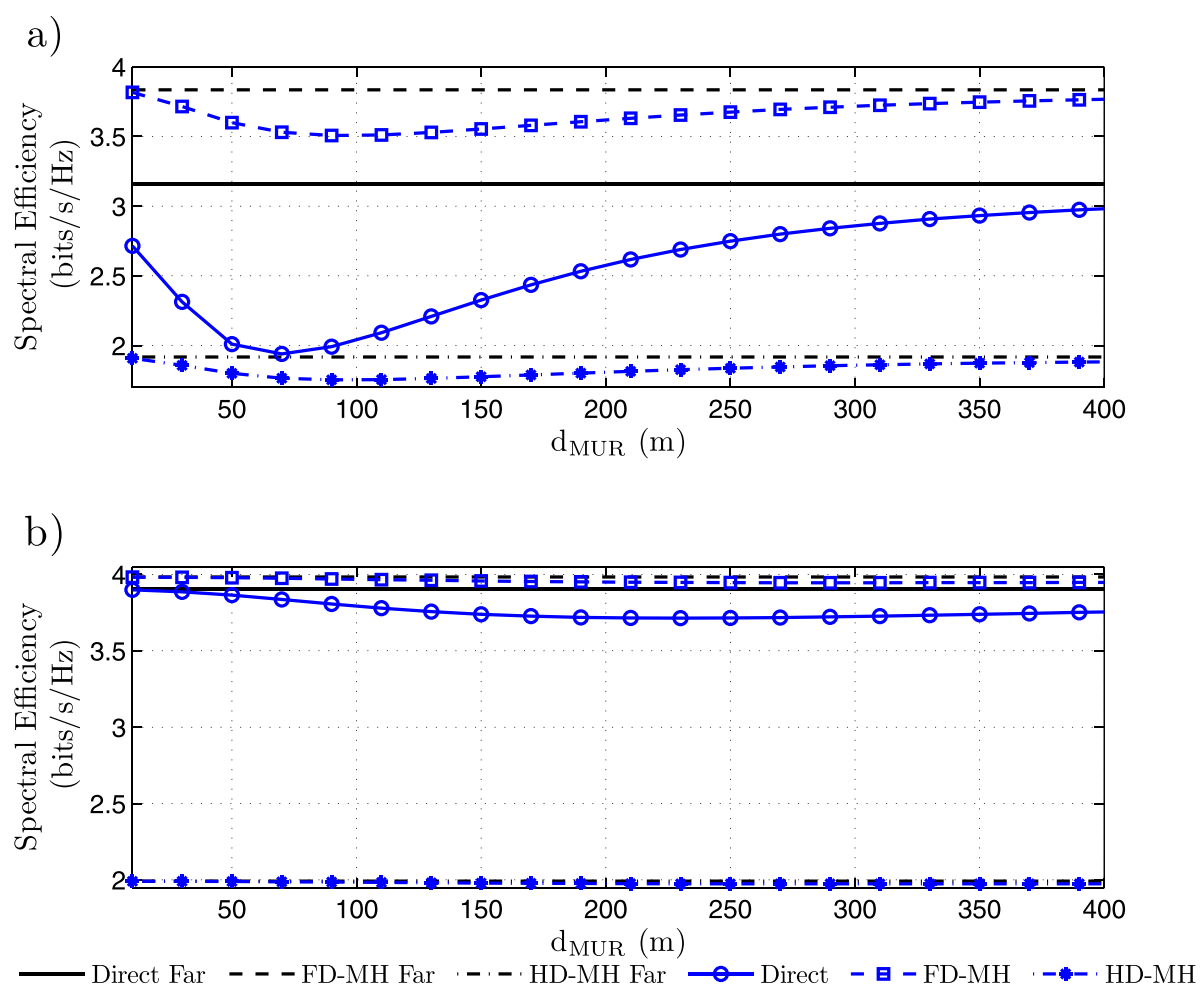

Figure 5 Spectral efficiency as a function of the $d_{M U R}$, assuming: (a) $\bar{\gamma}_{S D}=15 \mathrm{~dB}$ and (b) $\bar{\gamma}_{S D}=25 \mathrm{~dB}$.

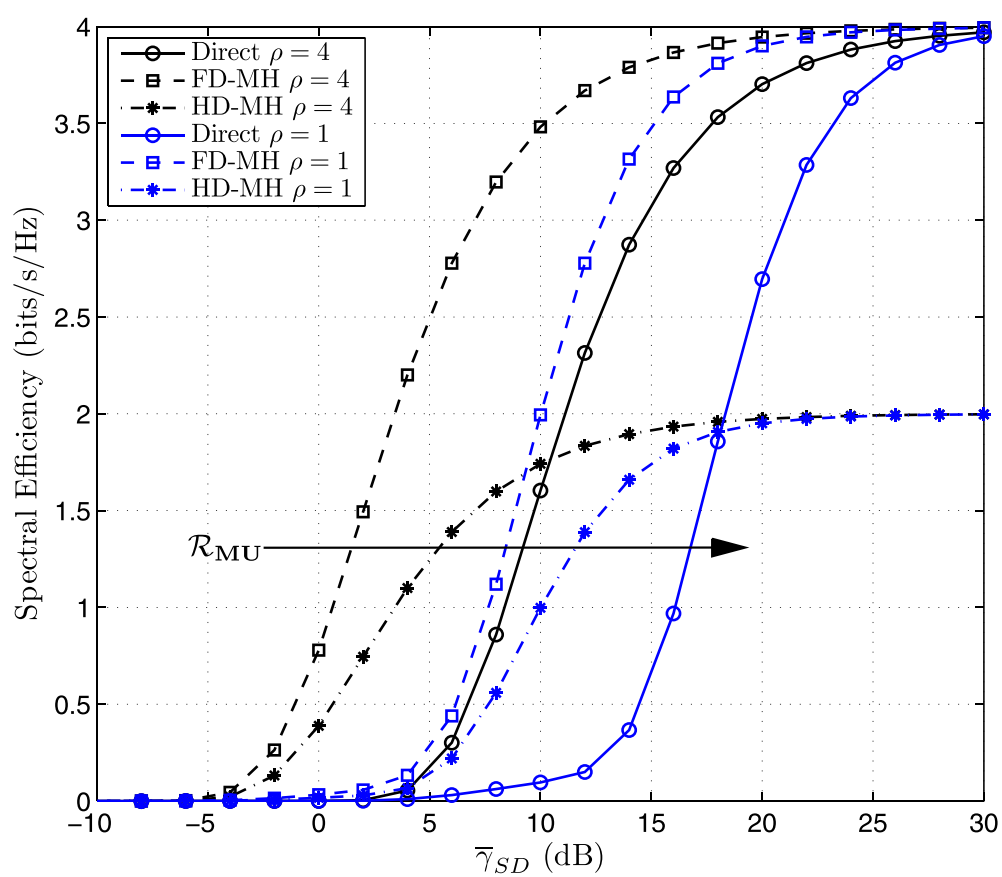

Figure 6 Spectral efficiency as a function of the $\bar{\gamma}_{S D}$, considering that $\rho=\frac{\mathcal{R}}{\mathcal{R}_{M U}}=[4 ; 1]$. 


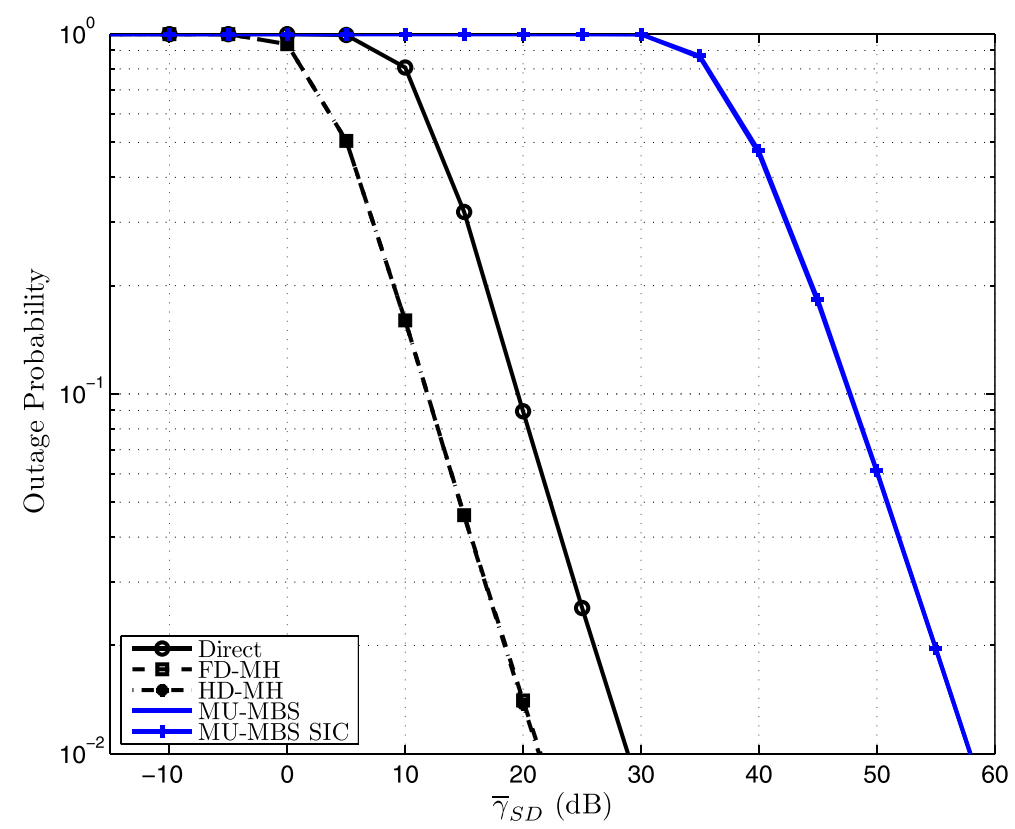

Figure 7 Outage probability as a function of the $\bar{\gamma}_{S D}$ with $\Phi=-40 \mathrm{~dB}$ and $\boldsymbol{d}_{M U R}=\boldsymbol{d}_{M U D}=\mathbf{1 0} \mathrm{m}$. We compare the performance of the MBS with and without interference. SIC is applied at the MBS to treat the interference caused by $S$ and $R$.

Figure 6 compares the femtocell spectral efficiency as a function of the $\bar{\gamma}_{S D}$ supposing different rates for the $M U$. Therefore, we assume that the information rate of the $M U$ may be $\mathcal{R}_{M U}=[1 ; 4]$ bits $/ \mathrm{s} / \mathrm{Hz}$, while the femtocell operates with a fixed rate $\mathcal{R}=4 \mathrm{bits} / \mathrm{s} / \mathrm{Hz}$. Thus, the ratio is defined as $\rho=\frac{\mathcal{R}}{\mathcal{R}_{M U}}=[4 ; 1]$. We also assume that
$d_{M U R}=10 \mathrm{~m}$ and $d_{M U D}=22.4 \mathrm{~m}$. As we can see from the figure, the performance of the femtocell is inversely proportional to the rate used by the $M U$. That is because it is easier to remove the $M U$ interference by means of SIC if $\mathcal{R}_{M U}$ is smaller. Moreover, note again that, besides solving problems related to the HD constraint, e.g., the problem

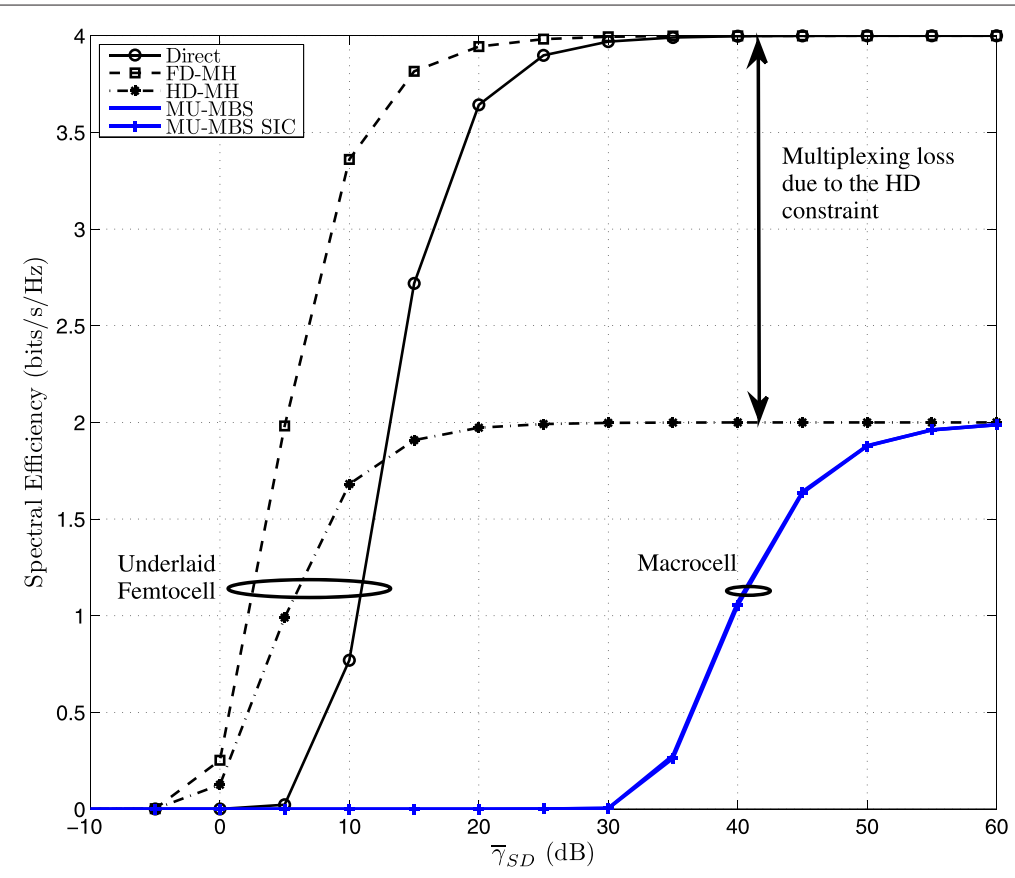

Figure 8 Spectral efficiency as a function of the $\bar{\gamma}_{S D}$ with $\Phi=-40 \mathrm{~dB}$ and $d_{M U R}=d_{M U D}=10 \mathrm{~m}$. 
of the hidden node, and the problem of multiplexing loss, FD relaying also allows the nodes within the femtocell to operate with much higher spectral efficiency than in the case of HD relaying, or with a considerable SNR advantage over the DT. This conclusion holds for any of the $M U$ rates considered in Figure 6.

Finally, we now analyze the performance of $M U$ at the MBS. First, Figure 7 shows the outage probability at the femtocell and at the MBS. We assume two cases: (i) the MBS sees the interference as additional Gaussian noise; (ii) the MBS applies SIC at the interference coming from $S$ and $R$. We conclude that the performance of the MBS remains almost the same as in the case without interference. In order to support our conclusion we recall that in Figure 5 we discussed that SIC performs better when the users are very close or very far from the receiver. Thus, once we assume nodes are at the cell border the MBS can considerably remove the interference. Moreover, in Figure 8 we analyze the performance of both macrocell and underlaid femtocell in terms of throughput. The performance at the MBS remains almost unaltered. Figure 8 also compares the performance of the HD and FD relaying schemes. As we can see in high SNR FD relaying achieves twice the throughput of the HD schemes. Such effect is caused because of the multiplexing loss introduced by the HD constraint which means that at least two slots are required for cooperation $[16,17]$. For instance, we observe that for a throughput of $1 \mathrm{bits} / \mathrm{s} / \mathrm{Hz}$ we obtain a $3-\mathrm{dB}$ gain in SNR between HD and FD, and more than $8 \mathrm{dBs}$ by using cooperative FD schemes instead of the DT.

\section{Conclusions}

In this article, we evaluate the performance of a heterogeneous network composed of a cooperative femtocell underlaid in a macrocell network. We consider a practical FD relay within the femtocell, which means that transmission and reception occur simultaneously and that self-interference is taken into account. We derive some closed-form expressions for the outage probability involving SIC, and we perform a semi-analytical performance analysis in terms of outage probability and spectral efficiency. We recall that by the usage of FD relaying we are able to solve problems related to the HD constraint, e.g., the problem of the hidden node, and the problem of multiplexing loss. Furthermore, our results show that FD relaying also allows the nodes within the femtocell to operate with much higher spectral efficiency than in the case of HD relaying, or with a considerable SNR advantage over the direct non-cooperative transmission.

\section{Endnote}

${ }^{\text {a }}$ Throughout this article the logarithm function is taken to base 2 unless stated otherwise.

\section{Competing interests}

The authors declare that they have no competing interests.

\section{Acknowledgements}

This study was conducted in the framework of the ICT project ICT-4-248523 BeFEMTO, which is partly funded by the EU.

Received: 21 November 2011 Accepted: 23 November 2012 Published: 22 December 2012

\section{References}

1. V Chandrasekhar, J Andrews, A Gatherer, Femtocell networks: a survey. IEEE Commun. Mag. 46(9), 59-67 (2008)

2. D Lopez-Perez, A Valcarce, GDL Roche, J Zhang, OFDMA femtocells: a roadmap on interference avoidance. IEEE Commun. Mag. 47(9), 41-48 (2009)

3. D Lopez-Perez, A Valcarce, GDL Roche, J Zhang, Intracell handover for interference and handover mitigation in OFDMA two-tier macrocell-femtocell networks. EURASIP J. Wirel. Commun. Netw. 2010(Paper ID 142629), 1-15 (2010)

4. Z Shi, MC Reed, M Zhao, On uplink interference scenarios in two-tiermacro and femto co-existing UMTS networks. EURASIP J. Wirel. Commun. Netw. 2010(Paper ID 240745), 1-8 (2010)

5. GDL Roche, A Valcarce, D Lopez-Perez, J Zhang, Access control mechanisms for femtocells. IEEE Commun. Mag. 48, 33-39 (2010)

6. HS Jo, P Xia, J Andrews, Downlink femtocell networks: open or closed? 2011 IEEE International Conference on Communications (ICC), (Austin, USA, 5-9 June 2011), pp. 1-5 doi:10.1109/icc.2011.5962618

7. P Xia, V Chandrasekhar, JG Andrews, Open vs. closed access femtocells in the uplink. IEEE Trans. Wirel. Commun. 9(12), 3798-3809 (2010)

8. X Chu, Y Wu, L Benmesbah, WK Ling, Resource allocation in hybrid macro/femto networks, 2011, IEEE International Conference on Communications (ICC), (Austin, USA , 5-9 June 2011),pp. 1-5

9. X Chu, JWu, H Wang, Outage probability analysis for collocated spectrum-sharing macrocell and femtocells. IEEE International Conference on Communications (ICC). (Austin, USA, 5-9 June 2011), pp. 1-5

10. M Shariat, A Quddus, R Tafazolli, Opportunistic spectrum reuse for femtocell networks, 2011 IEEE 73rd Vehicular Technology Conference (VTC Spring), (Budapest, Hungary, 15-18 May 2011), pp. 1-5 doi:10.1109/VETECS.2011.5956521

11. Z Bharucha, A Saul, G Auer, H Haas, Dynamic resource partitioning for downlink femto-to-macro-cell interference avoidance. EURASIP J. Wirel. Commun. Netw. 2010(Paper ID 143413), 1-12 (2010)

12. F Pantisano, K Ghaboosi, M Bennis, R Verdone, A self-organizing solution for interference avoidance in TDD underlay femtocells, 2010 Conference Record of the Forty Fourth Asilomar Conference on Signals, Systems and Computers (ASILOMAR), (Pacific Grove, USA, 7-10 Nov 2010), pp. 492-495 doi:10.1109/ACSSC.2010.5757607

13. F Pantisano, K Ghaboosi, M Bennis, M Latva-Aho, Interference avoidance via resource scheduling in TDD underlay femtocells, 2010 IEEE 21st International Symposium on Personal, Indoor and Mobile Radio Communications Workshops (PIMRC Workshops), (Istanbul, Turkey, 26-30 Sept 2010), pp. 175-179 doi:10.1109/PIMRCW.2010.5670356

14. CHM de Lima, M Bennis, K Ghaboosi, M Latva-aho, Interference management for self-organized femtocells towards green networks, 2010 IEEE 21st International Symposium on Personal, Indoor and Mobile Radio Communications Workshops (PIMRC Workshops), (Istanbul, Turkey, 26-30 Sept 2010) pp. 352-356 doi:10.1109/PIMRCW.2010.5670393

15. J Laneman, D Tse, G Wornell, Cooperative diversity in wireless networks: efficient protocols and outage behavior. IEEE Trans. Inf. Theory. 50(12), 3062-3080 (2004)

16. G Kramer, M Gastpar, P Gupta, Cooperative strategies and capacity theorems for relay networks. IEEE Trans. Inf. Theory. 51(9), 3037-3063 (2005)

17. R Tannious, A Nosratinia, Spectrally-efficient relay selection with limited feedback. IEEE J. Sel. Areas Commun. 26(8), 1419-1428 (2008)

18. H Alves, RD Souza, Selective decode-and-forward using fixed relays and packet accumulation. IEEE Commun. Lett. 15(7), 707-709 (2011) 
19. H Alves, RD Souza, G Fraidenraich, M Pellenz, Throughput performance of parallel and repetition coding in incremental decode-and-forward relaying. Wirel. Netw. 18, 881-892 (2012)

20. TRiihonen, S Werner, R Wichman, Z Eduardo, On the feasibility of full-duplex relaying in the presence of loop interference, IEEE 10th Workshop on Signal Processing Advances in Wireless Communications, 2009. SPAWC '09, (Perugia, Italy, 21-24 June 2009) pp. 275-279 doi:10.1109/SPAWC.2009.5161790

21. TRiihonen, S Werner, R Wichman, Optimized gain control for single-frequency relaying with loop interference. IEEE Trans. Wirel. Commun. 8(6), 2801-2806 (2009)

22. T Kwon, S Lim, S Choi, D Hong, Optimal duplex mode for DF relay in terms of the outage probability. IEEE Trans. Veh. Technol. 59(7), 3628-3634 (2010)

23. T Riihonen, S Werner, R Wichman, Comparison of full-duplex and half-duplex modes with a fixed amplify-and-forward relay, IEEE Wireless Communications and Networking Conference, 2009. (Budapest, Hungary, 5-8 April 2009) pp. 1-5 doi:10.1109/WCNC.2009.4917634

24. T Riihonen, S Werner, R Wichman, Hybrid full-duplex/half-duplex relaying with transmit power adaptation. IEEE Trans. Wirel. Commun. 10(9), 3074-3085 (2011)

25. M Duarte, A Sabharwal, Full-duplex wireless communications using off-the-shelf radios: feasibility and first results, 2010 Conference Record of the Forty Fourth Asilomar Conference on Signals, Systems and Computers (ASILOMAR), (Pacific Grove, USA, 7-10 Nov. 2010) pp. 1558-1562 doi:10.1109/ACSSC.2010.5757799

26. TM Cover, JM Thomas, Elements of Information Theory, 2nd edn. (Wiley-Interscience, Hoboken, NJ, 2006)

27. R Narasimhan, Individual outage rate regions for fading multiple access channels, IEEE International Symposium on Information Theory, 2007. ISIT 2007, (Nice, France, 24-29 June 2007) pp. 1571-1575 doi:10.1109/ISIT.2007.4557446

28. J Andrews, Interference cancellation for cellular systems: a contemporary overview. IEEE Wirel. Commun. 12(2), 19-29 (2005)

29. B Kaufman, E Erkip, J Lilleberg, B Aazhang, Femtocells in cellular radio networks with successive interference cancellation, 2011 IEEE International Conference on Communications Workshops (ICC), (Kyoto, Japan, 5-9 June 2011) pp. 1-5 doi:10.1109/iccw.2011.5963551

30. A Adhikary, V Ntranos, G Caire, Cognitive femtocells: breaking the spatial reuse barrier of cellular systems, Information Theory and Applications Workshop (ITA) 2011, (San Diego, USA, 6-11 Feb. 2011) pp. 1-10 doi:10.1109/ITA.2011.5743563

31. 3GPP, HNB and HNB macro propagation models. CMCC, CATT, Huawei, Qualcomm, Motorola, 3GPP TSG-RAN WG1 (2007)

32. $3 G P P$, Home node $b$ radio frequency RF requirements (FDD). Technical Specification Group Radio Access Networks, 3GPP TR 25.820 version 9.0.0 release 9, Technical Report (2009)

33. 3GPP, Channel models for femtocell. CMCC , CATT, Huawei, Qualcomm, Motorola, 3GPP TSG-RAN WG1 (2010)

34. T Riihonen, S Werner, R Wichman, Mitigation of loopback self-interference in full-duplex MIMO relays. IEEE Trans. Signal Process. 59(12), 5983-5993 (2011)

35. A Goldsmith, Wireless Communications. (Cambridge University Press, Cambridge, MA, 2005)

36. A Papoulis, SU Pillai, Probability, Random Variables and Stochastic Processes. (McGraw-Hill, New York, 2001)

37. A Ghosh, R Ratasuk, Multi-antenna systems for LTE eNodeB, 2009 IEEE 70th Vehicular Technology Conference Fall (VTC 2009-Fall), (Anchorage, Alaska, USA, 20-23 Sept 2009) pp. 1-4 doi:10.1109/VETECF.2009.5378666

38. V Chandrasekhar, M Kountouris, J Andrews, Coverage in multi-antenna two-tier networks. IEEE Trans. Wirel. Commun. 8(10), 5314-5327 (2009)

doi:10.1186/1687-1499-2012-365

Cite this article as: Alves et al.: Enhanced performance of heterogeneous networks through full-duplex relaying. EURASIP Journal on Wireless Communications and Networking 2012 2012:365.

\section{Submit your manuscript to a SpringerOpen ${ }^{\mathcal{O}}$ journal and benefit from:}

- Convenient online submission

- Rigorous peer review

- Immediate publication on acceptance

- Open access: articles freely available online

- High visibility within the field

- Retaining the copyright to your article

Submit your next manuscript at $\boldsymbol{\wedge}$ springeropen.com 